\title{
ANÁLISE DOS FATORES PROXÊMICOS NA COMUNICAÇÃO COM O PACIENTE LARINGECTOMIZADO
}

\author{
Namie Okino Sawada* \\ Márcia Maria Fontão Zago* \\ Cristina Maria Galvão* \\ Elaine Ferreira** \\ Elizabeth Barichello***
}

SAWADA, N.O.; ZAGO, M.M.F.; GALVÃO, C.M.; FERREIRA, E.; BARICHELLO, E. Análise dos fatores proxêmicos na comunicação com o paciente laringectomizado. Rev.latino-am.enfermagem, Ribeirão Preto, v. 8, n. 4, p. 7380 , agosto 2000 .

Esse estudo analisou os fatores proxêmicos das interações entre os profissionais da saúde e os pacientes laringectomizados no pós-operatório mediato. Utilizamos a técnica de observação não participante com roteiro pré-estabelecido. Os dados foram analisados à luz do referencial Teórico de Hall. O sexo e a cor dos interlocutores não influenciaram a distância mantida nas interações pelo número de elementos observados; a categoria assistência técnica de enfermagem foi a predominante; a postura adotada foi compativel com a finalidade dos encontros; o toque instrumental com contato visual foi predominante e o tom de voz foi adequado às distâncias mantidas. Ocorreram a presença de obstáculos em algumas interações, demonstrando que a comunicação ficou prejudicada.

UNITERMOS: proxêmica, pacientes, laringectomia; periodo pós-operatório

\section{INTRODUÇÃO}

Em nossa experiência profissional com pacientes laringectomizados, adquirida através da assistência de enfermagem prestada no período pré e pós-operatório, juntamente com os alunos de graduação em enfermagem e da participação do Grupo de Apoio e Reabilitação de Pessoas Ostomizadas - GARPO - Laringectomizados, freqüentemente deparamo-nos com dificuldades e relatos que evidenciam problemas na comunicação com o paciente laringectomizado após a cirurgia.

Segundo PARVULESCU ${ }^{14}$ o pessoal de enfermagem constantemente se depara com reações e emoções provenientes do cuidado com pacientes que sofreram cirurgias radicais como a laringectomia; ressalta que o trauma causado pela retirada de um órgão, a deformidade, a má aparência pela presença de tubos e curativos e a incapacidade para comer ou respirar normalmente representa um súbito impacto sobre o paciente e a família. A aceitação desse fato requer tempo e adaptações se fazem necessárias.
O paciente submetido a uma laringectomia total apresenta um problema imediato da perda da voz, ficando com a sua comunicação verbal oral prejudicada.

No processo de reabilitação do paciente laringectomizado, o ensino constitui o foco central. Para a consecução desse ensino, a comunicação não-verbal é um aspecto importante. Para BLONDIS \& JACKSON ${ }^{3}$ o laringectomizado apresenta um clássico exemplo de dinâmica de interação não-verbal, ou seja, após a cirurgia o paciente passa a comunicar-se exclusivamente de maneira não-verbal ou verbal escrito.

Apesar de $65 \%$ de toda a comunicação que ocorre em uma interação ser de caráter não-verbal ${ }^{7,8}$, sabemos que em nossa sociedade e principalmente em nossas atividades profissionais, a comunicação verbal é a mais difundida e valorizada. A comunicação não-verbal é mais demorada e requer que os interlocutores estejam atentos para esse tipo de comunicação.

O paciente laringectomizado tem medo de sufocação, falta de ar e geralmente manifesta esse medo através da comunicação não-verbal com: olhar suplicante,

Trabalho Subvencionado por Projeto Integrado de Pesquisa CNPq, processo $\mathrm{n}^{\circ}$ 520604/96.2

* Professor Doutor do Departamento de Enfermagem Geral e Especializada da Escola de Enfermagem de Ribeirão Preto da Universidade de São Paulo

** Bolsista de aperfeiçoamento do Projeto Integrado de Pesquisa $\mathrm{n}^{\circ}$ 520604/96.2

*** Bolsista de iniciação científica do CNPq - processo $\mathrm{n}^{\circ}$ 520604/96.2 
inquietação das mãos, tremores, expressões faciais tensas, inquietação, excitação, aumento dos movimentos respiratórios e pulso ${ }^{3}$.

É imprescindível que o enfermeiro entenda estes sinais não-verbais com o objetivo de diminuir a ansiedade do paciente e transmitir-lhe confiança. A forma como o pessoal da saúde se coloca diante desses pacientes, bem como a distância mantida e a maneira como realiza os procedimentos, refletem no como o mesmo se sente em relação ao outro. Muitas vezes, o pessoal da saúde demonstra repulsa, falta de aceitação e atitudes negativas com relação a esse tipo de paciente.

O uso do espaço é um meio de comunicação nãoverbal e influencia o relacionamento interpessoal. A proxêmica estuda o significado social do espaço, ou seja, estuda como o homem estrutura inconscientemente o próprio espaço.

O número de investigações acerca do modo como as pessoas utilizam o espaço vêm crescendo nas últimas décadas, inicialmente na área da antropologia e psicologia e hoje encontramos vários estudos na área da enfermagem.

A nível internacional podemos citar os trabalhos de ALLEKIAN $^{1}$; BARRON $^{2}$; HAYTER ${ }^{9}$; MINCKLEY ${ }^{10}$; SCOTT $^{18}$; STILLMAN $^{20}$ e TATE $^{21}$. No Brasil, ressaltamos os trabalhos de CARVALHO ${ }^{5,6}$; NODA et al. ${ }^{13}$; SAWADA \& GALVÃO ${ }^{15}$ e SAWADA ${ }^{16}$.

Todos esses estudos demonstram a importância de se observar as relações espaciais que deve haver entre o profissional de saúde e o paciente, tanto na abordagem física do paciente quanto no planejamento da divisão das enfermarias ou quartos.

Motivadas por essas considerações, propomo-nos a realizar essa investigação com a finalidade de verificar como o pessoal da equipe de saúde se comunica nãoverbalmente com os pacientes laringectomizados, logo após a cirurgia. Dessa forma o objetivo dessa pesquisa é:

- Analisar os fatores proxêmicos proposto por $\mathrm{HALL}^{8}$, durante as interações da equipe de saúde e pacientes laringectomizados no período pós-operatório mediato.

\section{REFERENCIAL TEÓRICO: A TEORIA PROXÊMICA DE HALL}

Edward T. Hall, antropólogo, foi pioneiro na conceitualização e estudo do espaço no relacionamento interpessoal. Utilizou o termo proxêmica para descrever a teoria do uso humano do espaço na comunicação.

A proxêmica estuda o significado social do espaço, ou seja, estuda como o homem estrutura inconscientemente o micro-espaço.
Segundo HALL ${ }^{8}$, a utilização do espaço é determinada culturalmente e a percepção da distância e a proximidade são resultados dos sistemas sensoriais (visual, auditivo, olfativo, tato). Em diferentes culturas, os canais sensoriais adquirem mais importância do que outros.

A proxêmica estuda o espaço em três aspectos: o espaço de características fixas (Ex.: paredes); o espaço de características semi-fixas (Ex.: disposição dos mobiliários, obstáculos e adornos); o espaço informal (o território pessoal ao redor do corpo do indivíduo).

$\mathrm{Na}$ cultura norte-americana, $\mathrm{HALL}^{8}$ identificou quatro distâncias interpessoais: distância íntima, distância pessoal, distância social e distância pública.

A análise proxêmica de $\mathrm{HALL}^{8}$ envolve oito fatores que compõem as suas categorias primárias:

1. postura-sexo: analisam o sexo dos participantes e a posição básica dos interlocutores (de pé, sentado, deitado).

2. eixo sociofugo - sociopeto: o eixo sociofugo demonstra o desencorajamento da interação enquanto o sociopeto implica no inverso. Essa dimensão analisa o ângulo dos ombros em relação à outra pessoa; a posição dos interlocutores (face-a-face, de costas um para o outro ou qualquer outra angulação).

3. cinestésicos: é o que provoca a proximidade entre os interlocutores. Analisa o contato físico a curta distância como o toque ou o roçar da pele e o posicionamento das partes do corpo.

4. comportamento de contato: este fator analisa as formas de relações táteis como acariciar, agarrar, apalpar, segurar demoradamente, apertar, tocar localizado, roçar acidental ou nenhum contato físico.

5. código visual: verifica o modo de contato visual que ocorre nas interações como o olho-no-olho ou ausência de contato.

6. código térmico: implica no calor percebido pelos interlocutores.

7. código olfativo: analisa as características e o grau de odor percebidos pelos interlocutores.

8. volume de voz: analisa a percepção dos interlocutores em relação ao espaço interpessoal.

\section{METODOLOGIA}

Antes de iniciarmos a coleta de dados, o projeto de pesquisa foi submetido à comissão de ética do hospital em estudo, tendo recebido parecer favorável para a realização da pesquisa. A coleta de dados foi realizada no período de setembro à novembro de 1997. No dia anterior à cirurgia, os pacientes eram contactados e informávamos sobre a pesquisa; após a sua aquiescência 
em participar, solicitávamos a assinatura no consentimento informado.

Para a coleta de dados utilizamos a técnica de observação direta não participante, com roteiro pré estabelecido (Anexo 1). O tempo de observação foi de no mínimo duas horas diárias por um período de três meses.

Os locais de observação foram as enfermarias de internação cirúrgica de um hospital governamental voltado para o ensino, pesquisa e assistência.

As observações foram registradas através de lápis e papel, segundo roteiro de observação. Registramos todas as interações ocorridas entre o pessoal da equipe de saúde e os pacientes laringectomizados no período pósoperatório mediato.

Os dados foram analisados à luz dos fatores proxêmicos de Edward T. HALL $^{8}$ exceto o código térmico e olfativo que através de nosso instrumento de observação não foi possível verificar.

Realizamos um banco de dados e utilizamos o programa Fox Pró 2 para a análise dos dados.

\section{RESULTADOS E DISCUSSÃO}

A caracterização dos elementos da saúde observados foram: 7 enfermeiros, sendo 3 do sexo masculino e 4 do sexo feminino; a faixa etária predominante foi de 30 à 40 anos (3), 40 à 50 anos (2), 20 à 30 anos (1) e mais que 60 anos (1); 5 deles eram brancos, 01 pardo e 01 mulato.

Os auxiliares de enfermagem que interagiram com os pacientes foram 29 , sendo 12 do sexo masculino e 17 do sexo feminino; a faixa etária predominante foi de 20 à 30 anos (11), 30 à 40 anos (8), 40 à 50 anos (8), 50 à 60 anos (1) e mais que 60 anos (1); 20 deles eram brancos, 4 pardos, 3 pretos e 2 mulatos.

Quanto as assistentes sociais foram um total de 3 , sendo todas do sexo feminino, na faixa etária de 40 à 50 anos, de cor branca.

Os médicos que interagiram foram 2 , e se encontravam nas faixas etárias de 20 à 30 anos e 30 à 40 anos, de cor branca.

Em relação a categoria de outros elementos, incluímos os alunos de graduação em enfermagem e auxiliares da nutrição, que foram um total de 6 elementos, dos quais 2 do sexo masculino e 4 do sexo feminino, nas faixas etárias entre 20 e 30 anos (2); 30 à 40 anos (1) e 50 à 60 anos (1); todos eram de cor branca.

No total de 20 pacientes, 17 (85\%) eram de cor branca, $2(10 \%)$ preta e $1(5 \%)$ parda; na faixa etária de 50 à 60 anos encontramos $9(45 \%), 5(25 \%)$ entre $40 \mathrm{e}$ 50 anos, 4 (20\%) de 60 à 70 anos e $2(10 \%)$ com mais de
70 anos; sendo que 19 (95\%) eram do sexo masculino e apenas $1(5 \%)$ do sexo feminino. A caracterização dos pacientes pode ser visualizada na Tabela 1 .

Tabela 1 - Distribuição dos pacientes observados, segundo faixa etária, sexo e cor.Ribeirão Preto, 1998

\begin{tabular}{c|c|c|c|c}
\hline Sexo e Cor & $\begin{array}{c}\text { Masculino } \\
\text { Branco }\end{array}$ & $\begin{array}{c}\text { Masculino } \\
\text { Pre to }\end{array}$ & $\begin{array}{c}\text { Fe minino } \\
\text { Parda }\end{array}$ & Total \\
Faixa Etária & - & - & - & - \\
\hline $\mathbf{2 0}$--- $\mathbf{3 0}$ & - & - & - & - \\
\hline $\mathbf{3 0}$--- $\mathbf{4 0}$ & - & - & - & 4 \\
\hline $\mathbf{4 0}$--- 50 & 4 & 2 & - & 10 \\
\hline $\mathbf{5 0}$--- $\mathbf{6 0}$ & 8 & - & - & 4 \\
\hline $\mathbf{6 0}$--- $\mathbf{7 0}$ & 4 & - & 1 & 2 \\
\hline $\mathbf{7 0}$---- & 1 & 2 & 1 & 20 \\
\hline Total & 17 & &
\end{tabular}

Esses dados corroboram com o levantamento do Ministério da Saúde, demonstrando que o câncer de laringe atinge predominantemente o sexo masculino, na faixa etária acima de 50 anos de idade ${ }^{4}$.

Segundo $\mathrm{HALL}^{8}$, o sexo é um dos fatores que interferem na distância escolhida durante as interações, entretanto não encontramos essa influência; atribuímos esse fato por termos observado situações profissionais técnicas e não sociais. Quanto a cor dos profissionais da saúde, $82,9 \%$ deles eram brancos. No estudo de NODA et al. ${ }^{13}$ ficou demonstrado que os negros olham mais para o interlocutor do que os brancos, quando interagem, porém em nosso estudo não foi possível detectar esse dado, visto que o número de negros foi de apenas 2,1\%.

As situações de interações ocorridas foram agrupadas nas seguintes categorias:

- Categoria 1: Avaliação e informações sobre as condições gerais do paciente;

- Categoria 2: Assistência técnica de enfermagem;

- Categoria 3: Ensino e comunicação verbal com o paciente;

- Categoria 4: Transporte ou mudança de posição do paciente.

Dentro da categoria 1: Avaliação e informações sobre as condições gerais do paciente - reunimos as situações em que o profissional avalia as condições gerais do paciente do tipo: avalia se o paciente está respirando bem, a presença de dor, as condições do local cirúrgico e do curativo, e quando esses elementos indagam o paciente sobre suas condições e/ou fornecem informações sobre a realização de procedimentos.

$\mathrm{Na}$ categoria 2: Assistência técnica de enfermagem - englobamos todos os procedimentos de enfermagem realizados com os pacientes como: 
instalação e interrupção de aerosol, verificação de sinais vitais, controle de gotejamento de soro, aspiração de traqueostomia, administração de medicação, administração de dieta por sonda nasoentérica, coleta de sangue, troca de frasco de soro e outros.

Na categoria 3: Ensino e comunicação verbal com o paciente - agrupamos as situações onde os profissionais da saúde conversam com os pacientes sobre as suas condições e sobre a sua família, além do ensino no autocuidado com a traqueostomia, e orientações sobre o GARPO (Grupo de Apoio e Reabilitação da Pessoa Ostomizada - laringectomizados).

$\mathrm{Na}$ categoria 4: Transporte ou mudança de posição do paciente - incluímos todas as situações de encaminhamento do paciente de um local para o outro, e as situações em que o pessoal auxilia no melhor posicionamento do paciente (mudança de decúbito).

Foram um total de 118 interações observadas, sendo que a categoria 2 - assistência técnica de enfermagem foi a mais freqüente, com 71 vezes $(60,1 \%)$, e as distâncias encontradas nessa categoria foram a íntima e pessoal; a categoria 1 - avaliação e informações sobre as condições gerais do paciente, foi a segunda de maior freqüência, com 37 vezes (31,3\%), e onde foram mantidas as distâncias íntima, pessoal e social. Em seguida, com 6 vezes $(5 \%)$ encontramos a categoria 4 - transporte ou mudança de posição do paciente, onde predominou a distância íntima. Por último, aparece a categoria 3 ensino e comunicação verbal com o paciente, com 4 $(3,3 \%)$ interações, onde observamos a distância pessoal.

Esses dados revelam que o predomínio das interações entre o pessoal da equipe de saúde e os pacientes laringectomizados no período pós-operatório mediato, estão relacionados com a execução de procedimentos $(60,1 \%)$ conforme demonstra a Tabela 2 .

Tabela 2 - Distribuição das situações de interação entre os profissionais da área da saúde e os pacientes laringectomizados, segundo a freqüência e a distância mantida. Ribeirão Preto, 1998

\begin{tabular}{l|c|c|c}
\hline \multicolumn{1}{c|}{$\begin{array}{c}\text { Situação de } \\
\text { interação }\end{array}$} & Frequência & $\begin{array}{c}\text { Distância } \\
\text { mantida }\end{array}$ & $\mathbf{N}^{\mathbf{0} \%)}$ \\
\hline $\begin{array}{l}\text { Avaliação e } \\
\text { informações sobre as } \\
\text { condições gerais do }\end{array}$ & 25 & Intima & $37(31,3 \%)$ \\
paciente & 2 & $\begin{array}{c}\text { Pessoal } \\
\text { Social }\end{array}$ & \\
\hline $\begin{array}{l}\text { Assistência técnica de } \\
\text { enfermagem }\end{array}$ & 61 & Intima & $71(60,1 \%)$ \\
\hline $\begin{array}{l}\text { Ensino e comunicação } \\
\text { verbal com o paciente }\end{array}$ & 4 & Pessoal & \\
\hline $\begin{array}{l}\text { Trarsporteou muckança } \\
\text { de posição do paciente }\end{array}$ & 6 & Intima & $6(3,0 \%)$ \\
\hline Total & 118 & & $118(100 \%)$ \\
\hline
\end{tabular}

Dentro das situações de assistência técnica de enfermagem, encontramos que a maioria das distâncias mantidas entre os profissionais de saúde e os pacientes laringectomizados, foi a distância íntima. Vários trabalhos ${ }^{1,2,5,16,18}$ já demonstraram que durante os procedimentos de enfermagem os profissionais adentram o espaço pessoal dos pacientes, por isso alertamos para que esses profissionais se conscientizem e evitem ao máximo a invasão desrespeitosa do espaço e a exposição desses pacientes, afim de minimizar as reações adversas como ansiedade, inquietação, luta e fuga. Alertamos, principalmente, na situação dos pacientes laringectomizados, que se encontram com sua auto-estima baixa e em condições de passividade devido a extensão da cirurgia e freqüência de procedimentos invasivos, como aspiração de traqueostomia, troca de frasco de drenos, punções de nível central, entre outros. Entendemos que esses pacientes, na situação descrita, reagirão através da ansiedade e inquietação, pois não estão em condições de lutar ou fugir.

Na categoria 1 - Avaliação e informações sobre as condições gerais do paciente, encontramos as distâncias íntima, pessoal e social. A distância íntima foi a mais freqüente, seguida da pessoal e social. Apesar da distância social estar presente em apenas 2 situações, entendemos que essa não seja a mais adequada para essas situações, pois não permite captar bem os sinais nãoverbais e o paciente laringectomizado comunica-se essencialmente através do não-verbal e verbal escrito.

$\mathrm{Na}$ categoria 4 - Transporte ou mudança de posição do paciente, como era de se esperar, a distância íntima prevaleceu nas seis situações observadas. Em estudo anterior ${ }^{16}$ encontramos relatos de pacientes que consideraram invasão do espaço pessoal a transferência deles sem informá-los, por isso ressaltamos a importância de informar aos pacientes para onde estão sendo encaminhados e, mesmo na mudança de decúbito, considerar a preferência de cada paciente.

$\mathrm{Na}$ categoria 3 - Ensino e comunicação verbal com o paciente, observamos que a distância mantida foi a pessoal, nas quatro situações levantadas. Na distância pessoal não ocorre o contato físico, apenas o visual, e acreditamos que nas situações de ensino dos pacientes laringectomizados essa distância seja adequada, pois permite um tom de voz baixo e uma visualização dos interlocutores; entretanto nas situações de comunicação verbal com esses pacientes, a distância íntima é a mais apropriada, visto que muitos sinais não-verbais são mais facilmente percebidos. Gostaríamos de ressaltar a pouca freqüência dessa categoria, o que demonstra que os elementos da equipe de saúde estão se comunicando verbalmente pouco com esses pacientes, e atribuímos esse resultado, à dificuldade de comunicação verbal que o paciente laringectomizado apresenta no pós-operatório. 
BLONDIS \& JACKSON ${ }^{3}$ caracterizam esse período como "O grito silencioso", onde o paciente, devido a edema facial extenso, pela retirada das cordas vocais e esvaziamento cervical, soroterapia e presença de drenos, comunica-se essencialmente pelo movimento palpebral gestual e expressão facial. A equipe de saúde necessita proporcionar meios que facilitem a comunicação desses pacientes, como a utilização da escrita através de lápis e papel ou o uso de lousa mágica.

Das 118 interações levantadas, a posição do pessoal da equipe de saúde em relação ao paciente foi predominantemente de pé, em apenas 02 situações a posição foi sentada, o eixo de contato, em sua maioria, foi frente à frente, somente em 04 casos o eixo foi lateral. A postura corporal, segundo $\mathrm{HALL}^{8}$, revela as características da relação estabelecida entre os interlocutores, e a posição frente à frente indica interesse mais restrito entre pessoas, seja negativo ou positivo, o posicionamento paralelo revela parceria e construção de objetivos comuns. Ao confrontarmos os eixos encontrados nesse estudo com as categorias de situações, detectamos bem essa definição, ou seja, em todas as situações de posicionamento frente à frente e lateral o pessoal de enfermagem estava interessado em executar alguma tarefa em prol do paciente.

Com relação ao tipo de contato, o tocar localizado se apresentou 14 vezes, o tocar localizado e acariciar ocorreram em 8 situações e apertar em 2 situações. A maioria dos toques presentes nas situações foi do tipo instrumental, sendo que somente em cinco situações o toque expressivo ocorreu. $\mathrm{O}$ trabalho de MONTAGU ${ }^{11}$ demonstra que as mulheres são mais comumente tocadas e tocam as pessoas com maior freqüência que os homens. Ao levantarmos o sexo do pessoal da equipe de saúde nas interações onde ocorreram o toque, encontramos: 8 situações envolveram o sexo masculino e 16 situações o sexo feminino, sendo que nas cinco situações onde ocorreu o toque expressivo, o sexo feminino prevaleceu. Esses dados vêm ao encontro do trabalho citado anteriormente e demonstra que a expressão de afetividade foi somente demonstrada pelo sexo feminino.

O toque instrumental, segundo SILVA ${ }^{19}$, se constitui no contato físico deliberado para o desenvolvimento de uma tarefa específica, enquanto que o toque expressivo ou afetivo ocorre espontaneamente e afetivamente, não estando necessariamente ligado a uma tarefa específica; geralmente é utilizado para demonstrar carinho, empatia, apoio, segurança e proximidade em relação ao paciente. A diferenciação dos tipos de toque ficou evidente ao cruzarmos os dados de interação em que ocorreram o toque expressivo com as categorias de interação; constatamos que essas se apresentaram somente nas categorias 1 - Avaliação e Informações sobre as condições gerais do paciente, e na categoria 3 - Ensino e comunicação verbal com o paciente, enquanto que o toque instrumental esteve presente na categoria 2 Assistência técnica de enfermagem. O trabalho de MULAIK et al. ${ }^{12}$ demonstrou que os pacientes perceberam que as enfermeiras dispensavam maior tempo com atividades relacionadas ao toque instrumental como: examinar, administrar medicação e ensinar.

Vários trabalhos ${ }^{3,11,12}$ têm demonstrado a eficácia do toque no tratamento de pacientes hospitalizados no que diz respeito à diminuição da ansiedade. Nessa perspectiva, ressaltamos a importância dos profissionais da saúde ficarem atentos a esse aspecto, visto que o paciente laringectomizado, no período pós-operatório, encontra-se com sua imagem corporal alterada (traqueostomia, edema), alteração da comunicação (perda da voz), e é constantemente submetido à procedimentos invasivos do tipo aspiração de traqueostomia, instalação de soro, colheita de sangue, dentre outros.

Durante a rotina hospitalar, observamos que a divisão do trabalho e a automatização na prestação de cuidados de enfermagem leva os profissionais a darem pouca importância ao toque. Assim, alertamos esses profissionais para utilizarem o toque instrumental aliado ao toque expressivo, permeando a assistência técnica, da expressão de afetividade e humanizando mais o cuidado prestado.

O contato visual ocorreu em 24 interações e estavam relacionadas àquelas em que ocorreram o toque. Esses dados corroboram com os trabalhos de NODA et

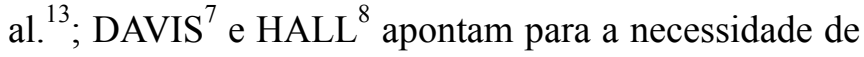
se observar as reações das pessoas ao serem tocadas, visto que a aceitação do toque envolve fatores que interferem na sua interpretação, como a cultura, o sexo, a idade e a raça dos interlocutores.

Quanto ao tom de voz do pessoal da equipe de saúde com o paciente laringectomizado encontramos em 10 situações o tom de voz baixo e o restante o tom normal. Ao relacionarmos o tom da voz baixo com a distância mantida, encontramos em todas elas a distância íntima, demonstrando que o tom de voz foi adequado às situações.

Ao analisarmos a presença de obstáculos dentro das interações, encontramos 10 situações com os seguintes obstáculos: o pessoal da equipe de saúde estava realizando outra atividade (2), suporte de soro entre os interlocutores (7) e o paciente estava apático (1). A presença de obstáculos durante as interações é um fator que interfere na comunicação, pois distancia ou cria barreira para o contato, por isso é importante que isso não ocorra. Dentre as situações que ocorreram a presença de obstáculos, encontramos as distâncias íntima, pessoal e social, sendo que na distância íntima encontramos uma barreira psicológica, ou seja, o paciente encontrava-se apático, recusando-se a interagir e, nas distâncias pessoal e social, observamos barreiras físicas como presença de 
suporte de soro e desenvolvimento de outra atividade por parte do pessoal da saúde.

Em estudo anterior ${ }^{17}$, sobre a forma de comunicação mais utilizada pelos laringectomizados totais, encontramos a mímica labial como a mais freqüente, apesar de não mais emitir a voz. A compreensão da mímica labial não é algo fácil e requer atenção, por isso alertamos os profissionais da saúde sobre esse aspecto que será dificultado muito mais, se houver a presença de barreiras.

\section{CONCLUSÕES}

Esse estudo permitiu-nos analisar os fatores proxêmicos das interações entre os profissionais da saúde e os pacientes laringectomizados no pós-operatório mediato. Consideramos de grande importância a análise desses fatores dentro do relacionamento com os pacientes laringectomizados, visto que nesse período eles se comunicam essencialmente pela comunicação não-verbal.

Diante dos objetivos propostos encontramos:

- A categoria profissional que mais interagiu com os pacientes foi o auxiliar de enfermagem(29), seguida dos enfermeiros(7), sendo a que menos interagiu, a dos médicos(2). Não detectamos influência do sexo e cor dos profissionais, no que diz respeito a distância mantida nas interações, pelo número de elementos observados.

- Das 118 interações analisadas, a categoria 2 - assistência técnica de enfermagem, foi a mais freqüente $(60,1 \%)$ com as distâncias íntima, pessoal e social, demonstrando que a maioria das interações estão relacionados com a execução de procedimentos; a distância íntima predominou dentro das situações e alertamos para que os profissionais fiquem atentos para o modo como adentram desrespeitosamente o espaço pessoal dos pacientes.

- Com relação a postura adotada pelos interlocutores encontramos o eixo frente a frente e lateral com maior freqüência, demonstrando que estes estão compatíveis com a finalidade dos encontros, ou seja, envolvem uma parceria com o paciente.

- Quanto ao tipo de toque, encontramos a maioria das vezes o toque instrumental, sendo que o toque expressivo ocorreu em apenas 5 situações e foram executados por mulheres. Esse dado corrobora com o trabalho de MONTAGU ${ }^{11}$, que demonstrou que as mulheres são mais comumente tocadas e tocam mais as pessoas.

- O contato visual ocorreu em 24 interações e esteve relacionado com a presença do toque, apontando para o fato de que, ao tocar, as pessoas necessitam observar as reações das outras com a finalidade de detectar a aceitação do toque.

- Com relação ao tom de voz do pessoal da equipe de saúde com o paciente larigectomizado, predominou o tom de voz normal e baixo, adequados com as distâncias mantidas nas interações.

- Ao analisarmos a presença de obstáculos, encontramos 10 situações com obstáculos, do tipo: presença de suporte de soro entre os interlocutores, o pessoal realizando outra tarefa e, em uma interação, o paciente encontrava-se apático. Esses obstáculos dificultam ou impedem a comunicação efetiva com o paciente laringectomizado total, visto que este já se encontra com a sua comunicação alterada.

\section{ANALYSIS OF PROXEMIC FACTORS IN THE COMMUNICATION WITH LARYNGOTOMIZED PATIENTS}

The present study analyzed the proxemic factors of interactions between health professionals and laryngotomized patients in mediate post-surgery. We used the non-participant observation technique with pre-established guidelines. Data were analyzed according to Hall's theoretical framework. The speakers' sex or color did not influence the distance maintained in the interactions; the nursing technical care category was predominant; the attitude adopted was compatible with the meeting goals; instrumental touch with visual contact was predominant, voice tone was adequate to the distances maintained and the authors observed the presence of obstacles in some interactions, which demonstrated that communication was impaired.

KEY WORDS: proxemic, laryngotomized, post-surgery

\section{ANÁLISIS DE LOS FACTORES PROXEMICOS EN LA COMUNICACIÓN CON EL PACIEN- TE LARINGECTOMIZADO}

Este estudio analizó los factores proxemicos de las interacciones entre los profesionales de salud y los pacientes laringectomizados en el postoperatorio mediato. Utilizamos la técnica de observación no participante con guía pre-establecida. Los datos fueron analizados a luz del referencial teórico de Hall. El sexo y color de los interlocutores no influenciaron la distancia mantenida en las interacciones, la categoría asistencia técnica de enfermería fue predominante; la postura adoptada 
fue compatible con la finalidad de los encuentros; el toque instrumental con contacto visual fue predominante y el tono de voz fue adecuado a las distancias mantenidas y ocurrieron obstáculos en algunas interacciones demostrando que la comunicación fue perjudicada.

TÉRMINOS CLAVES: proxemica, laringectomizado, postoperatorio

\section{ANEXO 1}

\section{Roteiro de Observação}

1. Dados de identificação do paciente

Nome:

Cor:

Idade:

Leito:

Sexo:

2. Dados de identificação do elemento de equipe de saúde:

2.1. Categoria: ( ) enfermagem
( ) médica
( ) nutricionista
( ) fonoaudiólogo
( ) outros - especifique
( ) enfermeiros
( ) técnico de enfermagem
( ) auxiliar de enfermagem
( ) atendente de enfermagem

2.2. Sexo:

2.3. Cor:

2.4. Idade:

3. Situação:

4. Observação da interação:
4.1. Tempo de duração :

4.2. Posição do elemento da saúde durante a intração
( ) de pé
( ) sentado
( ) outros. Especifique:

4.3. Distância
( ) intima $(0-0,50 \mathrm{~cm})$
( ) pessoal $(0,50-1,20 \mathrm{~m})$
( ) $\operatorname{social}(1,20-3,60 \mathrm{~m})$
( ) pública (acima de $3,60 \mathrm{~m}$ )

4.4. Tom de voz
( ) baixo (sussuro)
( ) normal (audível)
( ) alto (grito)

4.5. Eixo dos interlocutores
( ) frente a frente
( ) lateral
( ) costas

4.6. Comportamento de contato
( ) tocar localizado
( ) apertar
( ) acariciar
( ) outros. Especifique:

4.7. Contato visual ( ) sim ( ) não

4.8. Presença de obstáculos entre os interlocutores
( ) $\operatorname{sim}$
( ) não

Especifique:

\section{REFERÊNCIAS BIBLIOGRÁFICAS}

01. ALLEKIAN, C.I. Instrusions of territory and personal space: an anxiety-inducing factor for hospitalized persons: an exploratory study. Nurs.Res., v. 22, n. 3, p. 236-241, 1973.

2. BARRON, A. The righ to personal space. Nurs.Times, v. 86, n. 27, p. 28-33, 1990.

3. BLONDIS, N.M.; JACKSON, B.E. Nonverbal comunication with patients: back to the human touch. New York: John Wiley, 1982.

04. BRASIL. Ministério da Saúde. Secretaria Nacional de Assistência à Saúde. Instituto Nacional de Assistência à Saúde. Instituto Nacional de Câncer Coordenação de programas de controle do câncer - PRO-ONCO. Estimativa de incidência e mortalidade por câncer no Brasil, 1996. Rio de Janeiro: PRO-ONCO, 1996. 19p.

05. CARVALHO, D.V. Intrusão física e visual no espaço pessoal do paciente hospitalizado acamado. São Paulo, 1977. 98p. Dissertação (Mestrado) Escola de Enfermagem, Universidade de São Paulo.
06. CARVALHO, D.V. Necessidade territorial do paciente hospitalizado. São Paulo, 1987. 115p. Tese (Doutorado) - Escola de Enfermagem, Universidade de São Paulo.

07. DAVIS, F. A comunicação não-verbal. Trad. Antônio Dimas. São Paulo: E. Summus, 1979. 196p.

08. HALL, E.T. A dimensão oculta. Lisboa: Relógio D'agua, 1986.

09. HAYTER, J. Territoriality as a universal need. J. Adv. Nurs., v. 6, n. 2, p. 79-85, 1981.

10. MINCKLEY, B. Space and place in patient care. Am. J. Nurs., v. 68, n. 3, p. 510-516, 1968.

11. MONTAGU, A. Tocar: o significado humano da pele. São Paulo: Summus, 1988.

12. MULAIK, J.S. et al. Patients perceptions of nurses use of touch. West J. Nurs.Res., v. 13, n. 3, p. 306-323, 1991. 
13. NODA, K.S.; POLTRONIERI, M.J.A.; SILVA, M.J.P. Análise de fatores proxêmicos em situação de pós-operatório. In: Congresso Brasileiro de Enfermagem em Centro Cirúrgico, 1, São Paulo, 1995. Anais. São Paulo: Sociedade Brasileira de Enfermagem de Centro Cirúrgico, 1995. p. 3-10.

14. PARVULESCU, N.F. Care for the surgically speechless patient. Nurs. Clin. North Am., v. 5, n. 3, p. 517-525, Sept. 1970.

15. SAWADA, N.O.; GALVÃO, C.M. Comunicação nãoverbal: análise proxêmica em situações de préoperatória. In: Seminário Brasileiro de Comunicação em Enfermagem, 3, Ribeirão Preto, 1992. Anais. Ribeirão Preto: Escola de Enfermagem de Ribeirão Preto da Universidade de São Paulo, 1992. p. 205-219.

16. SAWADA, N.O. O sentimento do paciente hospitalizado frente à invasão de seu espaço territorial e pessoal. Ribeirão Preto, 1995. 196p. Tese (Doutorado) - Escola de Enfermagem de Ribeirão Preto, Universidade de São Paulo.
17. SAWADA, N.O.; ZAGO, M.M.F.; GALVÃO, C.M.; FERREIRA, E.; MARTINEZ, E.L. A comunicação após a laringectomia total. In: Carvalho,E.C. de. (Org). Comunicação em Enfermagem: relatos de pesquisas do $6^{\circ}$ Simpósio Brasileiro de Comunicação em Enfermagem. Ribeirão Preto: Fundação Instituto de Pesquisa em Enfermagem, 1998.

18. SCOTT, A.L. Human interaction and personal boundaries. J. Psychol. Nurs., v. 26, n. 8, p. 2327, 1998.

19. SILVA, M.J.P. Comunicação tem remédio: a comunicação nas relações interpessoais em saúde. São Paulo: Ed.Gente, 1996.

20. STILLMAN, M.J. Territoriality and personal space. Am. J. Nurs., v. 79, n. 10, p. 1670-1672, 1978.

21. TATE, J.W. The need for personal space in institutions for the elderly. J. Gerontol. Nurs., v. 6, n. 8, p. 439-449, 1980.

21. TATE, J.W. The need for personal space in institutions for the elderly. J. Gerontol. Nurs., v. 6, n. 8, p. 439-449, 1980. 